\title{
Authors*:
}

Dr Lydia Evans (1) (2)

Dr Ruth J. Tully (1) (3) (corresponding author)

(1) The University of Nottingham

Centre for Forensic and Family Psychology

Division of Psychiatry and Applied Psychology, School of Medicine

B06 YANG Fujia Building

Wollaton Road

Nottingham

NG8 1BB, UK

Tel: $+44(0) 1158466747$

Email: ruth.tully@nottingham.ac.uk

(2) Cambian Group, UK.

(3) Tully Forensic Psychology Ltd, Nottingham, UK

*Joint authorship. 


\section{The Triarchic Psychopathy Measure (TriPM): Alternative to the PCL-R?}

\section{Abstract}

Psychopathic personality disorder is the subject of many research papers and in particular in the context of forensic settings, where its link to risk of future violent has been established. This topic is well examined but there is still considerable debate bout the nature of the construct and how psychopathy is measured. Contemporary models such as the triarchic theory (Patricks, Fowles and Krueger (2009) have been put forward yet the research into psychopathy tends to rely on one assessment tool, the Psychopathy Checklist-Revised (PCL-R; Hare, 2003) that is argued not to capture elements of psychopathy such as boldness. The Triarchic Psychopathy Measure (TriPM; Patrick, 2010) is a measure that is based on the triarchic theory, and it places an equal focus on boldness when measuring psychopathy. It is however a self-report instrument, and this approach has many limitations. This paper aims to review the scientific support for the TriPM and to discuss its potential application to clinical practice. It concludes that the TriPM may not yet be a contender for the PCL-R throne as the sole tool of choice for psychopathy measurement, but the research into the application of the TriPM is expanding our understanding of psychopathy as a construct.

Key words: psychopathy, Triarchic Psychopathy Measure, PCL-R, personality disorder, psychological assessment, psychopathic personality disorder 
$\underline{\text { Introduction }}$

Psychopathic personality disorder is characterized by a constellation of interpersonal, affective and behavioral characteristics. Psychopathic traits overlap with anti-social/dissocial personality traits. Traditionally psychopathy is seen to comprise of an interpersonal/affective element (factor 1) and a social deviance (factor 2) (Hare, 1998), that has progressed to a more nuanced 4-factor model, based on sophisticated latent variable modeling with a diversity of large sample studies (Hare \& Neumann, 2008). There is a wealth of research that has been conducted via a range of psychopathy assessments. However, few scales have been examined with the rigor that is required to reliably use the scales in forensic settings, other than the Psychopathy Checklist-Revised (PCL-R; Hare, 2003), which is the most widely used and validated clinical diagnostic tool within criminal justice settings, and more recently the Self Report Psychopathy scale (Vitacco, Neumann \& Pardini, 2014; Tew, Harkins \& Dixon, 2015).

The PCL-R is divided into two factors. The first factor ('Factor 1') describes a constellation of interpersonal and affective traits commonly considered to be fundamental to the construct of psychopathy. The items in this factor are concerned with clinical inferences about affective processes and about verbal and interpersonal style. The second factor ('Factor 2') reflects those features of psychopathy associated with an impulsive, antisocial, parasitic and unstable lifestyle. Factor 2 therefore comprises items that are central to anti-social personality disorder (lifestyle and antisocial) and Factor 1 comprises items that are related to personality (interpersonal and affective). This 2 prong approach is useful, as it helps to break down the personality to understand it more comprehensively; for instance, one can consider if a patient's score is higher on the anti-social side or on the personality side of psychopathy. This can help guide treatment recommendations in relation to both the treatment pathway, and any responsivity needs within any treatment. Although he PCL-R is not a risk assessment tool in itself, psychopathy has been found to be a robust risk factor for future violence in a variety of diverse populations (Forth, Hart \& Hare, 1990; Hill, Rogers \& Bickford, 1996; Quinsey, Rice \& Harris, 1995; Rice \& Harris, 1992; Serin, 1991, 1996; Serin \& Amos, 1995). Thus, identification of psychopathic individuals is important in the criminal justice system because it guides risk management and treatment approaches to work with this sub group of offenders. 
Given the resources that may be put into managing those identified as psychopathic or as having high levels of psychopathic traits, if one is assessing a person tor psychopathy it is vital to do this correctly given the ethical and psycho-legal implications of doing so. The PCL-R has come under criticism for its focus on criminality and there is debate regarding what 'criminality' actually reflects with regard to the concept of 'criminality' not being in line with the developmental, longitudinal, structural and genetic research on psychopathy (see Hare \& Neumann, 2010; Skeem \& Cooke, 2010;). Its labour and time intensive administration has also been criticised (Copestake, Gray \& Snowden, 2011). Consequently, alternative measures such as self-report tools for the measurement of psychopathy are being empirically tested in the field. This current paper examines a relatively newly developed self-report psychopathy measure, the brief Triarchic Psychopathy Measure (TriPM; Patrick, 2010). This tool was developed to provide a framework to organise and clarify constructs related to psychopathy. This framework was outlined by Patrick, Fowles and Krueger (2009) who proposed three core constructs of psychopathy; meanness, boldness and dis-inhibition, which were incorporated into the TriPM. This review will cover the TriPM's scientific properties, implementation in research and its clinical application.

\section{Development of the Triarchic Psychopathy Measure (TriPM)}

The TriPM is a 58 item self-report measure consisting of three scales; meanness (19 items), boldness (19 items), and disinhibition (20 items). The measure uses a four point Likert scale with the response options 'mostly false', 'false', 'mostly true' and 'true'. The respondents are required to rate the degree to which each item applies to them on this scale. The tool was developed by Patrick (2010) in the USA and the measure and manuscript entitled "Operationalizing the Triarchic Conceptualisation of Psychopathy; Preliminary Description of the Brief Scales for Assessment of Boldness, Meanness and Disinhibition" is available online at www.phenxtoolkit.org. A significant limitation of the tool as it stands, is that the tool has not been peer-reviewed through academic journals or other scientific examination by experts, with the process of peer review being seen as a necessary part of quality control in the development of new tools. Whilst some peer-reviewed research has followed the development of the TriPM, some caution should be taken in reliance on the tool without further validation given this limitation in its 
development, and those using the tool should be aware of the research into its validity, as well as its strengths and limitations in clinical or research application.

The tool was developed from Patricks, Fowles and Krueger's (2009) triarchic conceptualisation of psychopathy. They highlighted the need to combine historical and more present-day perspectives (e.g. neuro-etiological perspectives) in understanding the psychopathic individual. Patrick, Drislane and Strickland (2012) emphasise that the triarchic conceptualisation is not a new or replacement theory to understand psychopathy. They instead suggest that current measures of psychopathy do, to varying degrees, index the three constructs proposed, but that measures such as the PCL-R do not capture distinct constructs. Specifically, they incorporate into their model a distinct and equal focus on boldness. Further, they argue that the PCL-R has a predominant focus on meanness rather than boldness and thus it can be suggested that the PCL-R fails to capture some important elements of psychopathy. The conceptualisation draws upon Cleckley's (1976) descriptive work with psychiatric patients, who developed sixteen diagnostic criteria to operationalize psychopathy. These criteria incorporate positive adjustment characteristics like absence of delusions and nervousness or suicidal intent, which links to the boldness domain of the triarchic model.

\section{Development of the TriPM meanness scale}

The meanness component of psychopathy is described in Patrick's 2010 manual as "reflecting tendencies toward callousness, cruelty, predatory aggression and excitement seeking" (p. 2). It is not clear from this how the excitement seeking element fits in the context of meanness, and this could be more indicative of boldness. The meanness scale was developed from items included in the Externalising Spectrum Inventory (ESI; Krueger, Markon, Patrick, Benning \& Kramer, 2007). The ESI is a 415 item self-report instrument which consists of 23 subscales. This measure is similar to the TriPM in that it was used to operationalize a particular model, specifically the Externalising Spectrum Model (Krueger, Hicks, Patrick, Carlson, lacono \& McGue, 2002). The ESI provides an assessment of disinhibitory problems and traits. It is important to note that this scale is also not yet widely validated (Venables \& Patrick, 2012). 
The TriPM meanness scale was constructed using the ESI's callous aggression sub-factor which operationalizes relational aggression, (low) empathy, destructive aggression, physical aggression, excitement seeking and honesty (lack of). Items related to empathy and honesty would be negatively scored. The brief meanness scale correlated highly with the callous sub-factor $(r=.65)$.

\section{Development of the TriPM disinhibition scale}

The triarchic conceptualisation describes disinhibition as "reflecting tendencies toward impulsiveness, irresponsibility, oppositionality and anger/hostility" (Patrick, 2010, p. 2). This scale is also derived from the ESI. Specifically, items were drawn from the following scales; irresponsibility, problematic impulsivity, theft, alienation, boredom proneness, impatient urgency, fraudulence, dependability, and planful control. The final brief disinhibition scale correlated with the ESI scales very highly $(r=.91)$. With correlations this high, suggesting that the scales are homogenous.

\section{Development of the TriPM boldness scale}

The boldness scale is a newly developed set of items by Patrick, Vaidyanathan, Benning, Hicks and Kramer (unpublished). The TriPM manual (Patrick, 2010) defines boldness as "nexus of high dominance, low anxiousness and venturesomeness" (p. 2). This scale was developed from the "fearless dominance" subscale of the Psychopathic Personality Inventory (PPI; Lilienfeld \& Andrews, 2006; PPI-R; Lilienfeld \& Widows, 2005). The PPI is also a self-report measure used to assess psychopathy. It consists of 187 items (the revised version is 154 items) across 8 overarching subscales that test fearless dominance, impulsive antisociality and coldheartedness. The PPI total score has been found to have convergent and criterion validity over 61 samples, (Miller \& Lynam, 2012). The fearless dominance scale was used as a template as it has demonstrated convergent validity with measures of behaviors indicative of boldness e.g. thrill seeking, for instance this element of the PPI was supported in its convergent and criterion validity by Miller and Lynam (2012). The TriPM boldness scale was developed into 3 facets with a total of 9 sub-facets; interpersonal behavior (persuasiveness, social assurance and dominance), emotional experience (resiliency, self-assurance, and optimism) and venturesomeness (courage, intrepidness and tolerance for uncertainty). The TriPM 
manual describes high correlations with Factor 1 of the PPI, which measures the "fearless" dominance behaviors ( $r=.77$ ) however Miller and Lynam's (2012) metaanalytic review found limited convergent validity and correlations inconsistent with many psychopathy conceptualisations. Although the boldness scale was originally developed from the fearless dominance scale of the PPI, it has since undergone significant revisions for its inclusion in the TriPM. It is therefore promising that it remains highly correlated, indicating that it may still measure the intended fearless dominance features. Although this fearless dominance subscale has been the PPI subscale that there have been more criticisms of with regard to validity (Gaughan et al., 2009), it is argued that the features that the PPl's fearless dominance measures are diagnostic specifiers of psychopathy, but not essential features of the disorder (Lynam \& Miller, 2012).

\section{Inter-correlations}

Inter-correlations within the TriPM indicate weak to moderate relationships between the three subscales of the TriPM (Patrick, 2010). These correlations could be explained by each TriPM scale being multidimensional, and these dimensions being differently associated, thus producing a statistical washout effect, rather than true low correlations or orthogonality (Neumann, Uzieblo, Crombez \& Hare, 2013). The triarchic model however is argued not to be based on a unitary or higher order construct of psychopathy like the PCL-R (Patrick, Drislane \& Strickland, 2012). It is argued that high co-variance is not expected because, as Patrick argues, the three scales should be measured as three separate constructs with intersecting components. This is similar to the PPI, as it does not require the subscales to fit a higher order concept and consequently the scales of this measure are un-correlated (Patrick, Fowles \& Krueger, 2009). However, as described below there is some overlap between the constructs, specifically meanness and disinhibition, that requires further consideration. For example, whether this is explained by the "intersecting" components, or whether this is an issue related to validity. As such it is not entirely clear and may indicate it is theoretically weaker than the PPI.

\section{Validity}

$\underline{\text { Concurrent validity }}$ 
Concurrent validity of tools is explored in order to establish how well the they correlate with other tests used to measure the same construct. Given the existing extensive research into the PCL-R and related Hare scales, it is not surprising that researchers have begun to compare other measures to the PCL-R, although in some cases such comparisons are highly questionable, for instance the findings of comparisons of the PPI to the PCL-R and PCL-SV (Malterer et al., 2010). The TriPM has been compared to other measures of psychopathy. These measures are expected to be strongly related to each other given that they are measuring similar constructs (Benning, Patrick, Salekin, \& Lestico, 2005). Much of the initial research with the TriPM has appeared to explore its concurrent validity as a way to validate the facets of psychopathy, although concurrent validity has also been found between the TriPM scales and various aspects of narcissism, empathy and normal range personality traits (Sellbom \& Phillips, 2013; Stanley, Wygant, \& Sellbom, 2013).

Patrick (2010) details correlations between the triarchic scales and the PCL-R in a sample of 148 male offenders. The triarchic scales demonstrated weak to moderate correlations with the PCL-R total scores $(r=.20-.32)$. The strongest correlations were observed between the disinhibition scale and the PCL-R. The boldness scale correlates significantly with the interpersonal facet of the PCL-R (argued to be the only facet that captures this element; $r=.27$ ) however this correlation was only modest. It has been highlighted that it could be argued that the absence of large correlations is to be expected, given the different measurement domains of a self-report measure and a clinical diagnostic tool such as the PCL-R (Malterer, Lilienfeld, Neumann \& Newman, 2010). However, development of the TriPM was also a way to conceptualise psychopathy in a different way to current tools such as the PCL-R. For example, Patrick et al. (2009) argue that the PCL-R does not fully capture the boldness element of psychopathy; therefore, it can be argued that correlations with the PCL-R should not be expected to be high as the construct being measured is conceptualised in a different way, and is therefore not the same construct. However, analysis of the sub-scales may be expected to show correlations, for instance it would be expected in Patrick's (2010) findings that higher correlations were observed between meanness and Factor 1 facet scores, as the PCL-R is argued to have more of a focus on meanness. However, these relationships were found to be weak. 
More recently, the TriPM was compared to the PCL-R in a sample of offenders (Venables et al., 2014). All three TriPM scales demonstrated significant modest to low moderate associations with a number of the PCL-R facets. Specifically, the Meanness scale was correlated with the PCL-R Interpersonal, Lifestyle, and Antisocial facets ( $r$ 's $=.24-.36$ ), although the Meanness scale (proposed to tap callous-aggression) fell short of significance in its association with the Affective facet $(r=.18 \mathrm{~ns})$. The Boldness scale was significantly associated with the Interpersonal and the Antisocial facets. The Disinhibition scale was the only scale to associate with only one PCL-R facet (i.e., Lifestyle). Surprisingly, none of the TriPM scales were correlated with the PCL-R Affective facet. Notably, the Meanness and Boldness scales were associated with a number of PCL-R facets, suggesting that, similar to the PCL-R (Neumann, Hare \& Newman, 2007) the TriPM scales may reflect a broad (super-ordinate) dissociality factor. In further support of this interpretation, most of the TriPM scales were no longer associated with the PCL$\mathrm{R}$ facets once the common variance shared among these scales were accounted for. One exception to this general finding was that the Boldness scale remained significantly associated with the Interpersonal and Antisocial PCL-R facets once the other TriPM scales were accounted for, which highlights that the Boldness scale may tap a broad (interpersonal-antisocial) dissocial construct, in-line with research on the PCL-R. In light of these findings, and the lack of specificity of the TriPM scales, the notion that the TriPM provides distinct scales is questionable when considering concurrent validity.

Correlations with other tools have been considered. The TriPM manual also describes correlations observed with other self-report psychopathy measures in a mixed gender sample of 94 students. The correlations were much higher when compared to those found with the PCL-R studies described above. This may be accounted for by the difference in measurement methods between the TriPM and PCL-R, for example self-report versus clinical interview/diagnostic tool. All three triarchic scales showed moderate to large associations with the PPI, Self-Report Psychopathy scale-III (SRP-III; Paulhus, Hemphill \& Hare, 2009), and the Youth Psychopathic Traits Inventory (YPI; Andershed, Kerr, Stattin \& Levander, 2002). The boldness scale had less success in its associations with the Levenson Self-Report Psychopathy scale (LSRP; Levenson, Kiehl \& Fitzpatrick, 1995) and did not demonstrate significant relationships. This may be due to the LSRP being based on 
similar facets to that of the PCL-R and therefore based on Patrick, Fowles and Krueger's (2009) argument it would not be expected to examine boldness. The TriPM appeared demonstrated its strongest relationships with the PPI. It can be suggested that this is an anticipated finding, given that elements of the PPI were used to develop the TriPM. Promisingly all three TriPM scales did show reasonable relationships ( $r=$ moderate and above) with the SRP-III and YPI, which have been developed independently of the triarchic model.

Stanley, Wygant and Sellbom (2013) examined correlations between the Triarchic Psychopathy Measure and the PPI-Short Form (Lilienfeld \& Hess, 2001) with 141 male and female prisoners. They report that the PPI-SF total score was significantly correlated to all Triarchic domains with moderate to high correlations. There was overlap between TriPM meanness/disinhibition, as they both related to the PPI impulsive antisociality scale. This may be expected given this PPI factor captures a range of subscales related to both mean and impulsive traits (e.g. Machiavellian egocentricity and carefree nonplanfulness).

More recently, Sellbom and Phillips (2013) report on how the TriPM performs against the PPI and LSRP in a female correctional sample. They also examined the Antisocial Processes Screening Device-Youth Version (APSD; Frick \& Hare, 2001) and the Inventory of Callous-Unemotional Traits (ICU; Frick, 2004) with their college sample. In the forensic sample they found that the TriPM scales had positive associations with other measures of psychopathy. TriPM boldness associated solely with the PPI (including fearless dominance), which could be explained by the LSRP and APSD scales having limited focus on boldness traits like the PCL-R. The TriPM meanness scale showed large correlations with the PPI total $(r=.64)$ and the subscale Machiavellian egocentricity $(r=.67)$. More moderate correlations were seen between meanness and the PPI's coldheartedness scale $(r=.38)$ which would be expected to be a stronger relationship based on the underlying theory of the subscales. This association was much lower than correlates found between meanness and self-centred impulsivity $(r=.58)$, which was not anticipated to correlate as highly. The relationship found between self-centred impulsivity and the disinhibition scale of the TriPM $(r=.60)$ was in fact very similar. Again not hypothesised but observed in the findings was the disinhibition scale displaying large 
associations with Machiavellian egocentricity $(r=.52)$. This was expected to be predominantly related to the meanness scale.

These unpredicted findings may be accounted for by the overlap found between disinhibition and meanness. Both the meanness and disinhibition scales were derived from the ESI, and so this may account for overlap or explained by the "intersecting" elements of the three domains Patrick, Drislane and Strickland (2012) proposed. However, this does not fit with the triarchic model's conceptualisation of "distinguishable" constructs.

Finally, Marion, Sellbom, Salekin, Toomey, Kucharski and Duncan (2012) developed a three factor model from a factor analysis that they performed on the TriPM, LSRP and PPI. The findings suggest that the analysis loaded onto three factors; disinhibitory and impulsive personality characteristics, mean or cold-hearted interpersonal style and a bold/fearless personality. They argue this supports the triarchic domains suggested by Patrick et al. (2009). This could be argued to also map onto Eysenck's 3 factor model of personality (Eysenck, 1982); neuroticism, extroversion and psychoticism. Those low in neuroticism would be characteristic of a low anxiety, bold personality. High scorers for extroversion would be the impulsive disinhibited personality type and finally high psychoticism would be indicative of the cold, aggressive and mean personality type. This could offer support to the theoretical underpinnings of the triarchic domains however as the factor analysis in Marion et al. (2012) was conducted at scale level, rather than item level, given that the TriPM subscales may well be multidimensional and not unidimensional, this may have resulted in a mis-specified model (see Neumann et al., 2013).

Overall, the findings suggest when compared to other psychopathy measures the TriPM appears to be measuring traits relevant to psychopathy and performing well against validated measures of psychopathy such as the PCL-R and PPI. Caution should be taken however, as some of the measures implemented are not typically "benchmark" tests, and therefore not commonly used in the forensic field. Further to this, a notable limitation is that some of the evidence for this is yet to be published and peer-reviewed, in particular the TriPM measure itself. Finally, further exploration of the overlap between meanness and disinhibition is needed.

\section{Discriminant Validity}


Discriminant validity tests whether constructs argued to be unrelated are in fact found to be unrelated. Stanley, Wygant and Sellbom (2013) evidence discriminant validity for the TriPM. They report non-significant beta weights for the scales that should be unrelated to the PPI-Short Form (PPI-SF). For instance, they argue that the PPI-SF cold-heartedness scale correlates as expected with meanness, but has negligible ability to predict boldness and disinhibition. They also highlight that PPI-SF fearless dominance has significant beta weights with boldness, but again it is unrelated to meanness and disinhibition. Selbom, Wygant and Drislane (2015) later found strong discriminant validity with triarchic model measure counterparts, namely the PPI and the PPI-Revised, when applied to non-clinical and male prisoner samples.

In Sellbom and Phillip's (2013) study a correlational analysis was performed for a female correctional sample, which compared the brief triarchic scales and psychopathy-relevant personality traits. This showed some evidence of discriminant validity. However, they also found some unexpected relationships between boldness and "experience seeking", which is anticipated to correlate more highly with disinhibition. Additionally, meanness showed further unexpected significant correlations with sensation seeking, disinhibition and boredom susceptibility, with which disinhibition was expected to correlate more highly with. This evidence suggests that perhaps some elements of the scales are not discriminating between distinct facets or constructs.

In conclusion, this suggests that meanness is also measuring disinhibitory tendencies. This could be explained by the inclusion of excitement seeking, and therefore measurement of low fear in the development of this particular scale. It raises the question as to whether this scale is capturing what could be considered a pure form of meanness. Whilst the model indicates that low fear plays a role in the meanness component of the model, it could be diluting the callous and predatory elements of this scale.

More evidence of discriminant validity between the TriPM and other tools is emerging in various samples (e.g. Crego \& Widiger, 2014) and this research should be expanded upon in order to provide a solid foundation for the furthering of the triarchic theory. Studies in this area should be planned and theoretically sound 
comparisons such as the results from a multitrait-multimethods matrix, to add to knowledge in this specific area.

\section{Content validity}

Content validity indicates whether a measure is adequately testing what it is intended to measure, in this case how well the TriPM is measuring psychopathic traits. The TriPM has been developed as a means to test the core constructs underpinning the psychopathic personality. Patrick, Fowles and Krueger (2009) developed the triarchic conceptualisation as they argued that current measures such as the PCL-R did not capture all relevant content associated with psychopathy e.g. boldness. Therefore, by developing this new measure Patrick (2010) has given consideration to empirical issues in the field and aimed to incorporate empirically relevant domains.

There remains some controversy in the field with researchers questioning the role of fearless dominance (boldness) in understanding psychopathy (Marcus, Fulton \& Edens, 2012; Miller \& Lynam, 2012). Specifically, it has been put forward that fearless dominance cannot be a central component to psychopathy. Some researchers have also argued that the concept of disinhibition is not unique to psychopathic groups (see Skeem, Polaschek, Patrick \& Lilienfeld, 2011). Nevertheless, Patrick, Drislane and Strickland (2012) put forward that the presence of disinhibition alongside boldness and meanness is enough to warrant identification of psychopathic traits, although conceding that disinhibition alone does not constitute a diagnosis of psychopathy.

These issues aside, initial support for the TriPM's content validity can be drawn from the findings for concurrent validity (described above) in studies such as Marion et al. (2012) and Stanley, Wygant and Sellbom (2013). They highlight how the TriPM scales are related to other measures of psychopathy relevant traits.

\section{$\underline{\text { Incremental Validity }}$}

Incremental validity assesses whether a new measure can add more predictive power than existing measures. Only one study by Stanley, Wygant and Sellbom (2013) reports findings for the incremental validity of the TriPM. The results positively showed the TriPM added incrementally beyond the PPI-SF to the 
prediction of criterion measures included in the study such as PPI-SF and the Narcissistic Personality Inventory (NPI; Raskin \& Terry, 1988). As this is the only study to have explored the TriPM's incremental validity, this is an area that may benefit from further exploration.

\section{Reliability}

$\underline{\text { Internal consistency }}$

Internal consistency reliability is used to test the cohesion of items in a single measure. The TriPM has shown some evidence of internal consistency. In their correctional sample, Sellbom and Phillips (2013) found that Cronbach's alphas for all three domains were .89 (boldness), .90 (meanness) and .89 (disinhibition). Stanley, Wygant and Sellbom (2013) indicate in their study alphas ranged from .77 (boldness), .84 (disinhibition) and .88 (meanness). Patrick (2010) does not report any reliabilities, neither do Marion et al. (2012). The ESl's internal consistencies from which the meanness and disinhibition subscales were developed ranged from .63 to .91. However, as discussed earlier, the ESI has not been validated, therefore it is problematic to rely on these findings without further empirical testing.

Kline (1999) highlights that acceptable values for reliability are .7 or higher, however reliabilities of .9 are considered too high. For example, as detailed above, Sellbom and Phillips (2013) report an alpha coefficient of .90 for meanness. This has implications for the subscales and overall measures validity. If reliability is too high this could indicate the meanness scale is too specific and lacks breadth in measuring the construct, and therefore could limit the TriPM's validity.

Sijtsma (2009) highlights caution in the use of Cronbach's alpha as a means to establish internal consistency. Sijtsma's paper discusses the limitations of alpha being a lower bound, so therefore underestimates reliability. Further, it highlights that alpha is not a measure of internal consistency, as there is no evident relationship between alpha and how cohesive a test is, as such it can tell us little about unidimensionality. Sijstma argues for the use of alternative tests that provide a greater lower bound (see Ten Berge \& Sočan, 2004) which when reported alongside alpha may encourage more appropriate approaches to estimating reliability. 
Although this is a statistical argument, relevant to any test development, this is relevant to consider when examining the TriPM.

Overall, internal consistency is important to consider for the TriPM because the TriPM scales may or may not be unidimensional. Thus, the TriPM should be examined for scale homogeneity (i.e. mean inter-item correlations). From the available research, the necessary item-level latent variable analyses have yet to be employed to test the internal construct validity of the TriPM.

\section{Test-retest reliability}

Test-retest reliability examines the degree of association between two sets of scores given by a respondent at different times. This tests the reliability and stability of a measure over time. There have been few reported studies of the test-retest reliability of the TriPM measure, and therefore, gaps remain in this area. One paper reports good test-retest reliability (Blagov et al., 2015) however this particular type of validity needs further examination. Without measuring the test-retest reliability of the TriPM it limits the extent to which the standard error of measurement can be established. For example, it is not possible to establish the extent to which an individual's score on the TriPM is inaccurate without guidance on how dispersed these scores can be for this population. Sijtsma (2009) highlights how administering a test to a random sample of participants once, does not tell researchers about individual propensity distributions, and how accurate a test is. It is therefore probable, by conducting testretest reliability, it reduces the standard error and provides more information about the individual.

In conclusion, research has focused on the validation of the TriPM measure, as this in turn provides evidence for the triarchic conceptualisation of psychopathy. Therefore, less focus has been placed on the reliability of the measure. However as discussed, reliability is inextricably linked to the measures validation so more focus is needed in this area, without this, findings produce a rather biased picture in relation to the tool. Given that reliability is one of the most important aspects of psychometric testing (Rust \& Golombok, 1999) it will be important for future research to examine this. 


\section{Clinical Application}

Clinical application is relevant to the assessment of psychopathy, as practitioners seek to use psychopathy measures to guide decision making in relation to treatment, management and resources needed for this client group. The PCL-R is the predominantly used as the main tool for assessing psychopathy; self-report measures are not typically used for this purpose.

Self-report tools are economical, easy to administer, enable one to examine response styles, and also eliminate issues related to inter-rater reliability (Lilienfeld \& Fowler, 2006). A further strength for the administration of self-report measures is the elimination of judgement errors by clinicians and the complexities of using inferences in diagnostic tools such as the PCL-R. It also removes the necessity to assess interrater reliability. The $\mathrm{PPI}$ is an example of a self-report tool that has grown in reputation and has been labelled the "gold standard self-report psychopathy measure" (Witt, Donellan \& Blonigen, 2009). Whilst the benefits in time, resources and training costs of developing a self-report measure that successfully tests psychopathy would be beneficial, practitioners may be reluctant to rely solely upon a self-report measure to accurately capture whether psychopathic disorder is present or not. Practitioners may be worried about self-report bias, impression management techniques, client insight into condition or other factors that may influence an offender's responses on a given day. If the client is a forensic patient or a prisoner, they may be reluctant to admit to behaviors that others may see as an indicator of ongoing or increased risk, thus underreporting symptoms of psychopathy.

Lilienfeld and Fowler (2006) highlight the difficulties that those who have high level so psychopathy may have in reflecting on their psychological problems given they may lack insight. They may also find it difficult to comment on the absence of their emotional experiences. The most common problem referred to in the literature is the deceitful and manipulative character of psychopaths, and the impact this has on outcomes on self-report measures. Cleckley (1976) referred to the psychopathic individual as being unable to be truthful. Consequently, self-report scales have come under criticism for the possibility of respondents to malinger (Lilenfeld \& Fowler, 2006). It may have been useful to add validity scales to the TriPM like those used in the PPI to assess the degree to which a respondent is consistent in their responses and how much they may falsify their responses. Miller, Jones and Lynam (2011) 
argue there is limited empirical support to indicate that psychopaths choose to malinger. They found that, in their sample of psychopathic individuals, they were able to accurately reflect and report on their personality providing that this had no direct impact on them. However, in practice this is often not the case; an assessment of psychopathy can have long-term implications for the offender. Marion et al. (2012) found that psychopathic individuals were no better at avoiding detection of malingering compared to non-psychopathic individuals when completing self-report measures. This may add weight to the use of self-report measures; however, they argue for inclusion of a test of response bias like the PPI, which currently the TriPM lacks. Ray et al. (2013) examined the issue of psychopathy and malingering, and their review raises important considerations about the differences between the study of self report measures for research purposes, and the use of such measures in high-stake situations such as Court assessments. This supports a recommendation for caution when using self-report psychopathy measures, even where a validity scale is included.

Whilst it appears that the TriPM's development was to generate empirical evidence for the triarchic model, its use as a tool in clinical settings is also relevant. Despite the limitations associated with self-report measures, there are some positive aspects to the development of this particular scale in terms of clinical practice issues. A strength of the TriPM is that it is brief to administer, taking approximately 15 minutes. It is easy to score in practice with some reverse-scored items. When scored, the user subsequently obtains 3 scores, one for each domain. The items themselves are brief, and avoid over-complexity. For example, "I get scared easily" or "I can convince people to do what I want". One item could appear more ambiguous to a client e.g. "I don't stack up well against most others". This could allude to physical stature or generally as a person. An item that may be useful to elicit an accurate picture of the patient is "others have told me they are concerned about my lack of self-control', because the respondent may be more able to highlight what others have told them, as opposed to reflecting on themselves. One limitation of the measure is the allocation of 4 visual symbols for the response options that could mislead a respondent, for example "mostly true" appears to look like a snowflake and "false" is similar to the symbol for male gender. The symbols in themselves may influence responses and in the current authors' views may well be a distraction to respondents rather that a useful aid. 
Following the administration and scoring of the tool, three scores are produced. However, there is no current guidance on cut-offs or descriptors for these domain scores to determine categorical diagnoses, it is solely based on higher scores being indicative of psychopathic features. Whilst the tool is developed for research purposes, in practice for clinicians deciding on treatment pathways for clients this may present issues in particular for those that might score moderately across the measure. Hare (2003) describes the complexity of developing appropriate cut-offs and the dangers of clinicians using cut off scores as definitive markers for whether an individual is psychopathic (for example scoring above 30). He highlights how criminal justice settings may use this as an arbitrary score which raises concerns about the individuals that may score 29 and therefore be identified as a non-psychopath. In this scenario confidence limits provide researchers and clinicians with a range of values which the relevant population value will fall within. As the TriPM is further utilised in research, it will allow for normative data to be collated to establish what the ranges may be. This could allow for descriptive guidance to be developed, for example, ranging from "low" to "very high". As it stands, it is unlikely the TriPM will be able to perform well as a diagnostic tool without further guidance on interpretation of the scores and this therefore hinders clinical-decision making based solely on the TriPM.

It is promising that the validation studies described in this review have been implemented with both genders, and in a range of samples (such as forensic and non-clinical community), especially given that the tool is yet to be fully examined. Comparative tools like the PCL-R were developed using predominantly male samples (Hare, 1991) and the PPI has been developed in community samples (Lilienfeld \& Andrews, 1996), therefore validation of the TriPM in a wide range of samples will add further clarity to the conceptual issues in the field, provide the development of a range of norms, and has the potential to offer a universal measure that is suitable for use with both genders.

The development and validation of the triarchic constructs has also influenced possible treatment approaches for this population. Patrick, Drislane and Strickland (2012) propose some initial concepts such as feedback-based response modification and attentional re-training. Development in this area is vital given the continuing perception in services that the psychopathic individual is untreatable. 
A self-report tool is nomothetic in nature, and therefore clinicians may be reluctant to rely solely on self-report as this will not capture the collateral information that one can learn about an individual through case files. Given some of the limitations highlighted above this may apply to the TriPM. Consideration may be given to supplementary assessments such as clinical interviews and scales that test response bias or malingering.

Malterer, Lilienfeld, Neumann \& Newman (2010) suggest the use of selfreport measures like the PPI to complement the PCL-R, as this tool can capture the positive adjustment features of psychopathic personality that the PCL-R does not. The TriPM could be implemented in a similar manner, however the continued reliance on the PCL-R despite its limitations will not serve to resolve the conceptual issues in the field. Whilst the TriPM may offer a useful framework by which to test the triarchic model it has its own limitations as a long standing measure for the identification of psychopathy. It is clear that the resolution of conceptual issues will be needed in order to provide clarity in terms of assessment approaches.

\section{Conclusion}

Conceptual debates are ongoing in relation to the concept of psychopathy, for instance with some authors arguing that boldness explains a key difference between psychopathy and antisocial personality disorder (Wall, Wygant \& Selbom, 2015) and the proposition that the PCL-R does not measure distinct constructs (Patrick, Drislane and Strickland, 2012). These arguments may not yet be resolved, however the development of tests based on contemporary theories is crucial to our assessment of personality, which in the forensic field may link to risk of harm. The triarchic model has support with regard to its utility as a framework (Drislane, Patrick \& Güler (2014) and further research into how to measure psychopathy according to this model is of value to researchers and clinicians alike (see Patrick \& Drislane, 2015).

The TriPM measure of psychopathy is in its infancy in terms of its empirical testing, however this review demonstrates some promising findings relating to its validation, in particular when compared against well validated psychopathy assessments such as the PCL-R. However, a problem with the TriPM could be that it 
places emphasis only on Cleckley's conceptualisations of psychopathy, when he did not conduct large scale empirical research to validate his theories (see Hare \& Neumann, 2008; Neumann, Hare \& Johansson, 2012). A further problem is that it was built primary through a rational process, with limited external construct validity based simply on (error prone) manifest variable correlations. The TriPM has yet to be rigorously examined via the intensive latent variable analyses that Hare's scales, including the SRP to some degree, have survived. For instance, the SRP is strongly related to the PCL-R, has been used in forensic settings, has clear latent structure and correlates in meaningful ways with cognitive, neurobiological and other correlates od psychopathy (Neumann, Hare \& Pardini, 2015). Until we understand the internal construct validity and dimensionality of the TriPM, the external construct validity evidence is ambiguous. It is argued that sound theoretical and validation tests can be conducted on tests such as the TriPM (see Smith, McCarthy \& Zapolski, 2009), and should be conducted prior to considering a tool reliable for widespread application, especially in the criminal justice system where the stakes are high with regard to both liberty and public protection.

Clinically, the TriPM offers a more economical and time efficient assessment of psychopathy. A significant limitation highlighted in this review is that the tool and some of the research that has been generated from it is not yet published, and therefore has not been subjected to further examination through peer-review. This critique also highlights that further testing of discriminant validity is needed given the overlap identified between meanness and disinhibition. Examination of the reliability of the measure was also rarely commented upon. This is likely related to the use of the tool to validate core constructs underpinning psychopathy, as opposed to the measure itself, which may have resulted in a biased picture of the various qualities of the tool. Despite this, it is clear that further examining the reliability of the scales will strengthen research in this area and provide further empirical support for the use of the TriPM in clinical settings. A good example of this is where researchers have examined TriPM scales and their links to clinical issues and distress e.g. anxiety (Fanti et al., 2015) and our knowledge of the empirical correlates of the TriPM continues to expand. In the process of exploring reliability, consideration should be given by researchers to Sijtsma's (2009) discussion points as to the use of Cronbach's alpha. Practitioners need to be aware of the limitations of using self- 
report measures to measure psychopathy, which of course applies to any self-report measure, but especially psychopathy, where respondents may have their own reasons for producing a response bias or they may lack insight. Clinicians also need to be as aware of the current conceptual dilemmas related to psychopathy. The TriPM does not offer itself as a diagnostic tool and therefore in clinical settings could be seen to lack utility to guide treatment, service provision and risk management. As a result, clinicians should use the tool clinically with caution and supplement it with other measures of personality. The TriPM is not yet a contender for the throne on which the PCL-R sits as a tool of choice for many in clinical practice, however, as the triarchic the empirically supported view of the utility of the TriPM may increase.

\section{References}

Andershed, H., Kerr, M., Stattin, H., \& Levander, S. (2002). Psychopathic traits in non-referred youths: A new assessment tool. In E. Blaauw \& L. Sheridan (Eds.), Psychopaths: Current International Perspectives (pp. 131-158). The Hague: Elsevier.

Benning, S.D., Patrick, C.J., Salekin, R.T., \& Leistico, A.R. (2005). Convergent and discriminant validity of psychopathy factors assessed via self-report: $A$ comparison of three instruments. Assessment, 12, 270-289.

Blagov, P. S., Patrick, C. J., Oost, K. M., Goodman, J. A., \& Pugh, A. (2015). Triarchic Psychopathy Measure: Validity in Relation to Normal-Range Traits, Personality Pathology, and Psychological Adjustment. Journal of Personality Disorders. e-View Ahead of Print.

Cleckley, H. (1976). The mask of sanity (5th ed.). St. Louis, MO: Mosby.

Cooke, D.J., Hart, S. D., Logan, C., \& Michie, C. (2004). Comprehensive Assessment of Psychopathic Personality - Institutional Rating Scale (CAPPIRS). Unpublished manuscript. 
Copestake, S., Gray, N.S., \& Snowden, R.J. (2011). A comparison of a self-report measure of psychopathy with the psychopathy checklist-revised in a UK sample of offenders. The Journal of Forensic Psychiatry \& Psychology, 22(2), 169-182.

Crego, C. \& Widiger, T. A. (2014) Psychopathy, DSM-5 and a Caution. Personality Disorders: Theory, Research and Treatment, 5(4), 335-347.

Drislane, L.E., Patrick, C.J. \& Arsal, G. (unpublished). Validity of the Triarchic Psychopathy Measure: Relations with other Psychopathy Measures and Psychopathy-related Traits.

Drislane, L.E., Patrick, C.J. \& Güler, A. (2014). Clarifying the Content Coverage of Differing Psychopathy Inventories Through Reference to the Triarchic Psychopathy Measure. Psychological Assessment, 26(2), 350-362.

Fanti, K. A., Kyranides, M. N., Drislane, L. E., Olivier, C, F, \& Andershed, H. (2015) Validation of the Greek Cypriot translation of the Tricarchic psychopathy Measure, Journal of Personality Assessment, online first, DOI: 10.1080/00223891.2015.1077452

Forth, A. E., \& Hart, S. D., \& Hare, R. D. (1990). Assessment of psychopathy in male young offenders. Psychological Assessment: A Journal of Consulting and Clinical Psychology, 2(3), 342-344. doi: 10.1037/1040-3590.2.3.342

Frick, P.J. (2004). The Inventory of Callous-Unemotional traits. Unpublished rating scale, University of New Orleans.

Frick, P.J., \& Hare, R.D. (2001). The antisocial processes screening device. Toronto, ON: Multi-Health Systems.

Gaughan, E. T., Miller, J. D., Pryor, L. R., \& Lynam, D. R. (2009). Comparing two alternative models of general personality in the assessment of psychopathy: A test of the NEO PI-R and the MPQ. Journal of Personality, 77, 965-996.

Hare, R.D. (1998) Psychopathy, affect and behaviour. In Psychopathy: Theory, Research and Implications for Society (eds D. Cooke, A. Forth \& R. Hare), pp. 105-139. Dordrecht: Kluwer 
Hare, R.D. (1991). The Hare Psychopathy Checklist-Revised. Toronto: Multi- Health Systems.

Hare, R.D. (2003). Manual for the Revised Psychopathy Checklist (2nd ed.). Toronto, ON, Canada: Multi-Health Systems.

Hare, R.D., \& Neumann, C.S. (2008). Psychopathy as a clinical and empirical construct. Annual Review of Clinical Psychology, 4, 217-246.

Hare, R.D. \& Neumann, C.S. (2008). Psychopathy as a clinical and empirical construct. Annual Review of Clinical Psychology, 4, 217-246.

DOI:10.1146/annuarev.clinpsy.3.022806.091.452

Hare R.D. \& Neumann, C.S. (2010). The role of antisociality in the psychopathy construct: comment on Skeem and Cooke (2010). Psychological Assessment 22(2), 446-54. DOI:10.1037/a0013635

Hill, C.D., Rogers, R. \& Bickford, M.E. (1996). Predicting aggressive and socially disruptive behaviour in a maximum security forensic hospital. Journal of Forensic Sciences, 41, 56-59.

Kreis MK, Cooke DJ, Michie C, Hoff HA, Logan C. (2012). The Comprehensive Assessment of Psychopathic Personality (CAPP): content validation using prototypical analysis. Journal of Personality Disorder, 26, 402-413.

Krueger, R.F., Hicks, B.M., Patrick, C.J., Carlson, S., lacono, W.G., \& McGue, M. (2002). Etiological connections among substance dependence, antisocial behaviour and personality: Modelling the externalising spectrum. Journal of Abnormal Personality, 111, 411-424.

Krueger, R.F., Markon, K.E., Patrick, C.J., Benning, S.D., \& Kramer, M.D. (2007). Linking antisocial behaviour, substance use and personality: An integrative quantitative model of the adult externalising spectrum. Journal of Abnormal Psychology, 116, 654-666.

Levenson, M.R., Kiehl, K.A., \& Fitzpatrick, C.M. (1995). Assessing Psychopathic Attributes in a Non-institutionalised Population. Journal of Personality and Social Psychology, 68, 151-158. 
Lilienfeld, S.O., \& Andrews, B.P. (1996). Development and Preliminary Validation of a Self-Report Measure of Psychopathic Personality Traits in Noncriminal Populations. Journal of Personality Assessment, 66, 488-524.

Lilienfeld, S.O., \& Fowler, K.A. (2006). The Self-Report Assessment of Psychopathy: Problems, Pitfalls and Promises. In Patrick, C.J. (eds) Handbook of Psychopathy. New York: The Guildford Press, p107-132.

Lilienfeld, S.O. \& Hess, T.H. (2001). Psychopathic personality traits and somatization: Sex differences and the mediating role of negative emotionality. Journal of Psychopathology and Behavioural Assessment, 23, 11-24.

Lilienfeld, S.O., \& Widows, M. (2005). Psychopathic Personality Inventory-Revised (PPI-R) professional manual. Odessa, FL: Psychological Assessment Resources.

Lynam, D.R., \& Miller, J.D. (2012). Fearless dominance and psychopathy: A response to Lilienfeld et al. Journal of Personality Disorders: Theory, Research, and Treatment, 3, 341-353.

Malterer, M.B., Lilienfeld, S.O., Neumann, C.S., \& Newman, J.P. (2010). Concurrent Validity of the Psychopathic Personality Inventory with Offender and Community Samples. Assessment, 17(1), 3-15.

Marcus, D.K., Fulton, J.J., \& Edens, J.F. (2012). The two-factor model of psychopathic personality: Evidence from the Psychopathic Personality Inventory. Personality Disorders: Theory, Research, and Treatment, 3, 140154.

Marion, B.E., Sellbom, M., Salekin, R.T., Toomey, J.A., Kucharski, L.T., \& Dunan, S (2012). An examination of the association between psychopathy and dissimulation using the MMPI-2-RF validity scales. Law and Human Behavior, 37(4), 219-230.

Miller, J.D., Jones, S.E., \& Lynam, D.R. (2011). Psychopathic traits from the perspective of self and informant reports: Is there evidence for lack of insight? Journal of Abnormal Psychology, 120, 758-764. 
Miller J.D, \& Lynam D.R. (2012). An examination of the Psychopathic Personality Inventory's nomological network: a meta-analytic review. Journal of Personality Disorders: Theory, Research, and Treatment 3(3), 305-26. doi:10.1037/a0024567

Neumann C.S., Hare, R.D., \& Newman, J.P. (2007). The super-ordinate nature of the psychopathy checklist-revised. Journal of Personality Disorder, 21(2), 102-17.

Neumann, C.S., Hare, R.D., \& Johansson, P.T. (2012). The Psychopathy ChecklistRevised (PCL-R), Low Anxiety, and Fearlessness: A Structural Equation Modeling Analysis. Personality Disorders: Theory, Research, and Treatment. 4(2), 129-137. DOI:10.1037/a0027886.

Neumann C.S., Hare R.D., Pardini D.A. (2015). Antisociality and the Construct of Psychopathy: Data From Across the Globe. Journal of Personality, 83(6), 678-92. DOI:10.1111/jopy.12127

Neumann, C.S, Uzieblo, K., Crombez, G., \& Hare, R.D. (2013). Understanding the Psychopathic Personality Inventory (PPI) in terms of the unidimensionality, orthogonality, and construct validity of the PPI-I and -II. Personality Disorders: Theory, Research and Treatment, 4(1), 77-79/

Patrick, C.J. (2010). Operationalising the Triarchic Conceptualisation of Psychopathy: Preliminary Description of Brief Scales for Assessment of Boldness, Meanness and Disinhibition. Unpublished Manual.

Patrick, C.J. and Drislane, L.E. (2015), Triarchic Model of Psychopathy: Origins, Operationalizations, and Observed Linkages with Personality and General Psychopathology. Journal of Personality, 83, 627-643. DOI:10.1111/jopy. 12119

Patrick, C.J., Drislane, L.E., \& Strickland, C. (2012). Conceptualising Psychopathy in Triarchic terms: Implications for treatment. International Journal for Forensic Mental Health, 11, 253-266. 
Patrick, C., Fowles, D.C., \& Krueger, R.F. (2009). Triarchic conceptualisation of psychopathy; Developmental origins of disinhibition, boldness and meanness. Development and Psychopathology, 21, 913-938.

Patrick, C.J., Vaidyanathan, U., Benning, S.D., Hicks, B.M., \& Kramer, M.D. (unpublished). Redefining measurement of the fearless-dominance component of psychopathy: Development and validation of the Boldness Inventory. Manuscript in preparation.

Paulhus D.L., (1998) Paulhus Deception Scales (PDS). Toronto, ON: Multi-Health Systems.

Paulhus, D., Hemphill, J., \& Hare, R. D. (2009). The SRP-III. Toronto, ON: MultiHealth Systems.

Quinsey, V.L., Rice, M.E. \& Harris, G.T (1995). Actuarial prediction of sexual recidivism. Journal of Interpersonal Violence, 10, 85-105.

Ray, J.V., Hall, J., Rivera-Hudson, N., Poythress, N.G., Lilienfeld, S.O., \& Morano, M. (2013). The relation between self-reported psychopathic traits and distorted response styles: A meta-analytic review. Personality Disorders: Theory, Research, and Treatment, 4, 1-14. DOI:10.1037/a0026482

Rice, M.E. \& Harris, G.T. (1992). A comparison of criminal recidivism among schizophrenic and nonschizophrenic offenders, International Journal of Law and Psychiatry, 15, 397-408.

Rust, J., \& Golombok, S. (1999). Modern Psychometrics: The Science of Psychological Assessment (2nd Eds). London; Routledge.

Sellbom, M., \& Phillips, T.R. (2013). An Examination of the Triarchic Conceptualisation of Psychopathy in Incarcerated and Nonincarcerated Samples. Journal of Abnormal Personality, 122, 208-214.

Selbom, M., Wygant, D. B., \& Drislane, L. E. (2015). Elucidating the construct validity of the psychopathic personality inventory triarchic scales. Journal of personality Assessment 97(4), 374-381

Serin, R.C. (1991). Psychopathy and violence in criminals. Journal of Interpersonal Violence, 6, 423-431 
Serin, R.C. (1996) Violent recidivism in criminal psychopaths. Law and Human Behavior, 20, 207-217

Serin, R.C. \& Amos, N.L. (1995). The role of psychopathy in the assessment of dangerousness. International Journal of Law and Psychiatry, 18, 231-238.

Skeem, J.L., \& Cooke, D.J. (2010). Is criminal behaviour a central component of psychopathy? Conceptual directions for resolving the debate. Psychological Assessment, 22, 433-445.

Skeem, J.L., Polaschek, D.L.L., Patrick, C.J. \& Lilienfeld, S.O. (2011). Psychopathic Personality: Bridging the Gap between Scientific Evidence and Public Policy. Psychological Science in the Public Interest, 12(3), 95-162.

Smith G.T., McCarthy D.M., \& Zapolski TC. (2009). On the value of homogeneous constructs for construct validation, theory testing, and the description of psychopathology. Psychological Assessment, 21(3), 272-84. DOI:10.1037/a0016699.

Stanley, J.H, Wygant, D.B., \& Sellbom, M. (2013). Elaborating on the Construct Validity of the Triarchic Psychopathy measure in a Criminal Offender Sample. Journal of Personality Assessment, 95(4), 343-350.

Tew, J., Harkins, L., \& Dixon, L. (2015). Assessing the reliability and validity of the Self-Report Psychopathy Scales in a UK offender population, The Journal of $\begin{array}{llll}\text { Forensic Psychiatry \& Psychology 26(2), 166-184. } & \text { \& }\end{array}$ DOI:10.1080/14789949.2014.981565

Venables, N.C., \& Patrick, C.J. (2012). Validity of the Externalising Spectrum Inventory in a Criminal Offender Sample: Relations with Disinhibitory Psychopathology, Personality and Psychopathic Features. Psychological Assessment, 24, 88-100.

N. C. Venables, J. R. Hall and C. J. Patrick (2014). Differentiating psychopathy from antisocial personality disorder: a triarchic model perspective. Psychological Medicine, 44, 1005-1013. doi:10.1017/S003329171300161X.

Vitacco, M.J., Neumann, C.S., \& Pardini, D.A. (2014). Predicting Future Criminal Offending in a Community-Based Sample of Males Using Self-Reported 
Psychopathy. Criminal Justice and Behavior 41(3), 345-363. DOI: $10.1177 / 0093854813500488$

Wall, T.D., Wygant, D.B., \& Selbom, M. (2015). Boldness explains a key difference between psychopathy and antisocial personality disorder. Psychiatry, Psychology and Law, 22(1), 94-105. 